\title{
Diagnostic accuracy of computed tomographic angiography (CTA) in the management of aneurysmal subarachnoid haemorrhage $(\mathrm{SAH})$
}

\begin{abstract}
Aim: To assess the diagnostic accuracy of three dimensional computed tomographic angiography (3D-CTA) for the detection of cerebral aneurysm in the patients of spontaneous subarachnoid hemorrhage (SAH).

Methods: This prospective study extended from $1^{\text {st }}$ November 2007 to $1^{\text {st }}$ February 2010. All the patients of spontaneous SAH were included in the study. Plain computed tomography (CT) head followed by computed tomographic angiography (CTA) was done in all patients using multi-slice spiral CT scan. Results were analyzed by both Radiologist and the Neurosurgeons. The points considered were

$$
\begin{aligned}
& \text { i. The site of aneurysm. } \\
& \text { ii. In multiple aneurysms, which is the aneurysm that has bled? } \\
& \text { iii. The relationship of vessels to the aneurysm. }
\end{aligned}
$$

Results: We have found 60 ruptured aneurysms in total of 75 patients on which 3D-CTA was done. 58 aneurysms found on 3D-CTA were confirmed on intra-operative findings during surgery and were surgically managed. In our study, CTA have a sensitivity of $96.7 \%$ and specificity of $75.0 \%$ for detecting aneurysms.

Conclusion: Majority of patients with intracranial aneurysms could be operated solely based on pre- operative 3D-CTA data sets. DSA should be used as an additional diagnostic tool in 3D-CTA negative patients.
\end{abstract}

Keywords: intracranial aneurysm, subarachnoid hemorrhage (SAH), computed tomographic angiography (CTA)
Volume I Issue 3 - 2014

\section{Javeed I Zargar, Altaf U Ramzan, Abrar A Wani, Feroz Shaheen, Nayil K Malik, Furqan A Nizami,Anil Dhar \\ Department of Neurosurgery, Sheri- Kashmir Institute of Medical Sciences (SKIMS), India}

Correspondence: Furqan A Nizami, Department of Neurosurgery, Super-speciality Hospital, GMC, Jammu, (J\&K) India,Tel 9797635257, Email drnizamifurqan@yahoo.co.in

Received: July 17, 2014 | Published: July 26, 2014
Abbreviations: DSA, digital subtraction angiography; $\mathrm{SAH}$, subarachnoid haemorrhage; CTA, computed tomographic angiography; SPSS, statistical package for social sciences

\section{Introduction}

One of the most frequent causes of subarachnoid hemorrhage is ruptured aneurysm. ${ }^{1,2}$ The frequency of subarachnoid hemorrhage is approximately 11 per 100,000 persons in the United States. ${ }^{3}$ Approximately 28,000 people per year in North American sustain subarachnoid hemorrhage associated with aneurysmal rupture. ${ }^{4}$

Subarachnoid hemorrhage secondary to ruptured aneurysm is a critical clinical situation demanding a very skilled surgical and medical management. Until now cerebral digital subtraction angiography (DSA) has been used as the criterion standard for aneurysmal detection and planning of their microsurgical treatment. However, as the method for diagnosis of subarachnoid hemorrhage moved from lumbar puncture to computed tomography, it seems that method for diagnosis of intracranial aneurysms is also moving from digital subtraction angiography to computed tomographic angiography
(CTA), especially CTA with three dimensional reconstructions of images. $^{5-8}$

DSA has several disadvantages other then time delay, including invasiveness because of required arterial puncture and intra arterial catheter manipulation, high skill level to perform the procedure and relatively high cost. ${ }^{9-11}$

CTA provides anatomical information about the aneurysm and the normal cerebral vessels more quickly than DSA and needs less institutional resources regarding equipment, trained personal and organization. Schmid et al. ${ }^{12}$ reported the high accuracy of high resolution $\mathrm{CT}$ in the direct diagnosis of cerebral aneurysms. Recent advances in helical CT have enabled Neuro-radiologists to evaluate cerebral aneurysms in short time. ${ }^{13,14}$ Moreover, CTA is not associated with the significant patient risks other than those associated with administration of iodinated contrast media.

The more recent one i.e., 3D-CTA what we have used as diagnostic tool in our patients is a non-invasive, quick, and reliable method. The use of 3D-CTA will reduce the period of time between the diagnosis 
of SAH and the diagnosis of the aneurysm and thus make it possible to operate more rapidly in case of emergency.

\section{Methods}

In this prospective study, all the patients of spontaneous subarachnoid hemorrhage (SAH) who presented to our institute were included. Diagnosis of SAH was based on CT scan. Patients were administered Nimodipine (60mg 4hrly), analgesics, anticonvulsants, and supplemental intravenous fluids. All baseline investigations were done.

CT angiography was done in all patients. CT scanning was done using multi-slice spiral CT scan, 64slice, Siemens Somatom sensation (Siemens, Enlargen, Germany). The CT scan and CT angiogram were analyzed by both Radiologist and the Neurosurgeons. The following points were discussed:

i. What is the site of aneurysm?

ii. In multiple aneurysms, which is the aneurysm that has bled?

iii. What is the relationship of vessels to the aneurysm?

iv. Is there any need for repeat study or digital subtraction angiogram (DSA).

If quality of angiogram is not good then repeat study was done, whereas if the quality was good but CT scan was showing non perimesencephalic SAH or had equivocal results then digital subtraction angiogram (DSA) was done.

In patients where decision for DSA was made, selective three or four vessel angiogram was done and the necessary information was gathered. DSA again was analyzed by all the investigators. The patients were operated after the diagnoses of the aneurysm. Intraoperative findings were correlated to pre operation information gathered from the radiological data. Any discrepancies found were noted. The data was analyzed using Statistical package for social sciences 11.5 version (SPSS) statistical software. Sensitivity \& specificity of CT angiography was estimated. The question of whether DSA is necessary in patients of SAH was assessed.

\section{Results}

Out of 75 patients in our study, female $53(70.7 \%)$ outnumbered the male patients $22(29.3 \%)$. The age of patients ranged from 14 to 76 years, the youngest patient being 14 years of age whereas the oldest was 76 years of age. Mean age at presentation was 49.88 years. The most common symptom at the time of presentation was sudden onset severe headache $(100 \%)$. Meningism in the form of neck stiffness was present in most of the patients $(81.3 \%)$.

Majority of patients $50(66.6 \%)$ were admitted after 24 hours of ictus, where as $25(33.3 \%)$ presented within $24 \mathrm{hrs}$ of ictus. $36(48 \%)$ of patients were fully conscious and oriented at the time of admission i.e., with GCS of 15.17 (22.6\%) had GCS 13-14. 21 (28\%) had GCS 9-12 and only one (1.3\%) had GCS of 3-8 at admission.

$66.6 \%$ of patients were in good Hunt \& Hess Grades (I-II) at presentation. Majority of patients $48(64 \%)$ in our series had Fisher grade II SAH. $6(8 \%)$ had hydrocephalus at presentation whereas 13 $(17.3 \%)$ of the patients showed intra-ventricular hemorrhage.

86 aneurysms were diagnosed on CTA in a total of 75 patients, and middle cerebral artery (MCA) was the commonest site of origin (Table
1). $54(62.70 \%)$ patient's harbored only one aneurysm while rest had multiple aneurysms (Table 2). Majority of the aneurysms $(63.9 \%)$ were small in size i.e., $\leq 10 \mathrm{~mm}$ (Table 3 ). Total of 60 aneurysms were found in operated patients, clipping was done in $56(93.3 \%)$ and muscle wrapping in $4(6.6 \%)$ patients. Intra-operative rupture occurred in $4(6.6 \%)$. Out of 75 patients included in our study $9(12 \%)$ patients died. The various causes of death are shown (Table 4).

Table I Site of origin

\begin{tabular}{ll}
\hline Site of Aneurysm & $\mathbf{N}(\%)$ \\
\hline A Com & $23(26.7 \%)$ \\
DACA & $09(10.4 \%)$ \\
MCA(Proximal) & $09(10.04 \%)$ \\
MCA(Bifercation) & $24(27.9 \%)$ \\
MCA (Distal) & $01(1.16 \%)$ \\
P Com & $03(3.4 \%)$ \\
Intracavernous & $02(2.32 \%)$ \\
Supraclinoid & $06(6.97 \%)$ \\
Vertebral & $01(1.16 \%)$ \\
Basilar Top & $03(3.4 \%)$ \\
P ICA & $02(2.32 \%)$ \\
PCA & $01(I .16 \%)$ \\
ICA Bifurcation & $02(2.32 \%)$
\end{tabular}

Table 2 Number of aneurysm

\begin{tabular}{lc}
\hline & $\mathbf{N}(\%)$ \\
Single Aneurysm & $54(62.70 \%)$ \\
Two Aneurysms & $13(30.2 \%)$ \\
Three Aneurysms & $02(6.9 \%)$ \\
Total & $86(100 \%)$ \\
\hline
\end{tabular}

Table 3 Size of aneurysms

\begin{tabular}{ll}
\hline & $\mathbf{N}(\%)$ \\
\hline Small $(\leq 10 \mathrm{~mm})$ & $55(63.9)$ \\
Large $(>10-24 \mathrm{~mm})$ & $23(26.7)$ \\
Giant $(>25 \mathrm{~mm})$ & $08(9.3)$ \\
Total & $86(100)$ \\
\hline
\end{tabular}

Table 4 Cause of death in 9 patients

\begin{tabular}{ll}
\hline Cause of Death & $\mathbf{N}(\%)$ \\
\hline Pre operative Bleed & $2(2.6)$ \\
Post operative Infarct & $2(2.6)$ \\
Operation site hematoma & $\mathrm{I}(\mathrm{I} .3)$ \\
Respiratory distress & $\mathrm{I}(\mathrm{I} .3)$ \\
Intraoperative bleed & $\mathrm{I}(\mathrm{I} .3)$ \\
Poor neurological grade & $\mathrm{I}(\mathrm{I} .3)$ \\
Respiratory infection \& & $\mathrm{I}(\mathrm{I} .3)$ \\
Septicemia & $9(\mathrm{I} 2.0)$ \\
Total & \\
\hline
\end{tabular}




\section{Discussion}

In our study, female patients were more than male patients. (F 53: M 22). Literature also shows predominance of females as demonstrated by Hoh et al. ${ }^{15}$, Kassel et al. ${ }^{16}$ (1:1.6) \& Bonita and Thomson et al. ${ }^{9}$ (65 male, 115 females). Pechllivanis et al. ${ }^{17}$ \& Rosennorm et al. ${ }^{18}$ also observed a female preponderance in patients of SAH (M 32: F 68).

The maximum i.e., 22 patients in our study were in the age group 51-60 followed by 12 patients in the age group of 61-70. The mean age at presentation was 49.88 years. This is in concordance with other studies available in literature with most of their patients in the $5^{\text {th }} \&$ $6^{\text {th }}$ decade of life. ${ }^{12,16,19}$ In Inagawa et al. ${ }^{20,21}$ series more patients were in age range of 17-93 years. Maximum number of patients was in the 7 th decades of life. A significant number of our patients $17(22.7 \%)$ were found to be hypertensive. Hypertension was present in $21 \%$ of patients studied by Kassel et al. ${ }^{16}$ and $50 \%$ of patients in Framingham study. ${ }^{22}$

In our study most common location of aneurysm was MCA $(\mathrm{n}=34,39.1 \%)$ followed by ACOM $(\mathrm{n}=23,26.7 \%)$. Pechllivanis et al. ${ }^{17}$ in their group of 62 patients found ACOM aneurysms (26\%) to be commonest followed by MCA (19\%). Ender et al. ${ }^{23}$ found (38\%) of the aneurysms in ACOM area whereas, only (32\%) were found in MCA complex. Rest of the aneurysms was seen in a lesser number in their study. Le Roux et al. ${ }^{24}$ in study of 25 patients of SAH, found MCA aneurysms to be more i.e., 18 followed by three aneurysms at carotid bifurcation and two at ACOM.

In our study of 75 patients, the total no. of aneurysms found on 3D-CTA was 86 . Only $54(62.70 \%)$ patients had single aneurysm, $13(30.2 \%)$ patients had two aneurysms each whereas two patients had three aneurysms each (6.9\%). Uysal et al. ${ }^{25}$ in their study of 32 patients, 28 patients were found to have aneurysms while no aneurysm was detected in 4 cases. Krogi et al. ${ }^{26}$ in their study of 35 patients detected 47 aneurysms. Their six patients had two aneurysms each and three had three aneurysms each.

In our study majority of aneurysms $55(63.9 \%)$ were small in size, 23 aneurysms (26.7\%) were large in size where as only 8 aneurysms $(9.3 \%)$ were of giant size. Krogi et al. ${ }^{26}$ had 35 patients in their study $\&$ observed that 14 aneurysms were very small, 16 were small, 14 were medium and three were large. Liang et al. ${ }^{6}$ in their study by both $\mathrm{CT}$ angiography and conventional angiography detected 15 aneurysms in 14 patients. The maximum dimensions of the aneurysm on CT angiography were less than $3.0 \mathrm{~mm}$ in three patients, $3.0-5.0 \mathrm{~mm}$ in four patients and more than $5.0 \mathrm{~mm}$ in eight patients.

In the study by Nyil et al. ${ }^{27} 66.50 \%$ of patients were seen within $48 \mathrm{hrs}$ of onset of ictus. In a study from south Sweden ${ }^{18} 63 \%$ patients were seen within $24 \mathrm{hrs}$ of ictus. In our study only $25(33.3 \%)$ patients presented within $24 \mathrm{hrs}$. of ictus whereas majority of patients $44(58.6 \%)$ presented within 24 to $72 \mathrm{hrs}$. of ictus. Time interval between ictus \& admission is directly related to the awareness of disease among the general practioners and the travel time required to reach the hospital. The referral area to our hospital is quite wide and most of the patients have to come from far off hilly places by road only.

In our study of 75 patients, 71 patients $(94.7 \%)$ presented with single bleed whereas 2 patients $(5.3 \%)$ had two bleeds. Edner et al. ${ }^{23}$ found that out of 146 patients seen, 54(36.98\%) had presented with more than one bleed prior to admission. In one study by Ljunggren et al. ${ }^{11} 41 \%$ of patients were admitted in good grades, $17 \%$ in intermediate grades, $42 \%$ in poor grade. $55 \%$ of patients were admitted in good grade and $45 \%$ in poor grade in another series. ${ }^{19}$

In our study $31(41.3 \%)$ of patients were in Hunt \& Hess grade I, $19(25.3 \%)$ were in grade II, $10(13.3 \%)$ patients were in grade III, $14(18.7 \%)$ were in grade IV whereas only $1(1.3 \%)$ was in grade V. In this study 50(66.6\%) patients were in good grade which is in concordance with the study by Ljunggren et al. ${ }^{11}$ who had more than $40 \%$ of their patients in good grade. Majority $36(48 \%)$ of our patients were fully conscious i.e., with a GCS of 15, whereas $17(22.6 \%)$ of patients were having a GCS of 13-14. Only $1(1.3 \%)$ patient was in the GCS range of 3-8. Majority of our patients $48(64 \%)$ were having a Fisher grade II on CT. 20(26.7\%) of the patients had a Fisher grade IV on CT. Pechllivanis et al. ${ }^{17}$ in a study of 84 patients, 57 patients were in grade III, 15 patients in grade II, 7 patients in grade I and 5 patients were in Fisher grade IV.

Generally vascular neurosurgeons are divided into two groups, one group uses DSA as a diagnostic procedure established over a long time and CTA as an additional diagnostic method and the other group consists of neurosurgeons considering 3D- CTA without DSA sufficient for the detection of aneurysms and a diagnostic tool with promising future. In our study we wanted to settle the question, if CTA can replace DSA as primary diagnostic tool for the diagnosis of ruptured or un-ruptured aneurysms.

3D-CT angiography requires only a venous access and a small amount of iodine radiological contrast agent. The quick imaging modalities allow rapid diagnosis of aneurysms soon after the diagnosis of SAH because it can even be done during the same radiological session. The views of every side including views from the side and the bottom or top of the aneurysm can be obtained with 3D-CTA. Planning of possible surgical approaches can also be done beforehand.

Aneurysm surgery solely based on 3D-CTA data sets requires a high sensitivity for detecting intracranial aneurysms. 92\% of all patients in a study by Pechlivanis et al. ${ }^{17}$ could be operated solely based on 3D-CTA data sets, when they analyzed their records retrospectively. On analysis of our data we found that 60 ruptured aneurysms were seen on 3D-CTA. Per operatively 58 aneurysms were confirmed and treated exactly as they were seen on 3D-CTA. Two patients were over diagnosed on 3D-CTA, and out of these two patients diagnosis of ACOM aneurysm was made on 3D-CTA but on surgery only a mild dilatation of ACOM was seen and no definitive aneurysm could be seen. In another case two aneurysm- ACOM and right MCA Aneurysm were diagnosed on 3D-CTA but no MCA aneurysm was found in this patient. This makes CTA to have a sensitivity of $96.7 \%$ for detecting aneurysms.

We failed to detect aneurysms in four of our patients. Amongst them DSA also failed to reveal the aneurysm in 3 cases. DSA revealed an aneurysm in one of the 3D-CTA negative cases. Hence with 3D-CTA in our study a specificity of $75.0 \%$ was seen. In literature a wide variation in regard to sensitivity ( $70 \%$ to $100 \%)$ and specificity ( $50 \%$ to $100 \%$ ) of $3 \mathrm{D}-\mathrm{CTA}$ for aneurysm detection exists. ${ }^{8,28,29}$

We used 3D-CTA as the only diagnostic tool for treatment planning in majority of our patients. The percentage of patients who have been operated solely based on the 3D-CTA data ranges between $33 \%{ }^{30,31}$ up to $93 \%{ }^{32}$ We have no program for emergency surgery and patients are scheduled for surgery as early as possible after diagnosis disregarding the classical concepts of timing of surgery after SAH. 
Patients included in our study were surgically treated by clipping of the neck of the aneurysm or wrapping through a pterional, frontal or sub occipital approach and using standard microsurgical technique. In the pterional approach the sylvian fissure was widely opened in every patient and the entire course of intracranial carotid artery, middle cerebral artery and the anterior cerebral artery of the side of surgical approach were exposed. The anterior communicating artery complex and the exit of posterior communicating and anterior choroidal arteries were carefully explored. The evaluation of the surgery included confirmation of the 3D-CTA findings and the presence of any finding not suspected by $3 \mathrm{D}-\mathrm{CTA}$.

Hoh et al. ${ }^{15}$ reported about a similar protocol for using 3D-CTA as the single diagnostic method for treatment planning with a reported sensitivity of $100 \%$ for symptomatic aneurysms.

\section{Conclusion}

Various conclusions drawn from our study revealed a sensitivity of $96.7 \%$ for detecting aneurysms with 3D-CTA and a specificity of $75.0 \%$. CT angiography is noninvasive, quick to organize and to perform. It provides very valuable anatomical information. The three dimensional capability and ability to demonstrate the aneurysm on the source image are added advantages in pre operative planning and microsurgical management of the patients of intracranial aneurysms. In accordance with other authors we think that in near future 3D-CTA could replace conventional angiography in most cases. Majority of patients with intracranial aneurysms could be operated solely based on pre- operative 3D-CTA data sets. If 3D-CTA does not show an aneurysm in spite of strong suspicion of aneurysmal bleed on plain CT, Digital subtraction angiography should be used as an additional diagnostic tool.

\section{Acknowledgments}

None.

\section{Conflicts of interest}

The authors declare there are no conflicts of interest related to the article.

\section{References}

1. King JT. Epidemiology of aneurysmal subarachnoid hemorrhage. Neuroimaging Clin N Am. 1997;7(4):659-668.

2. Rinkel GJ (2003) Treatment of patients with aneurismal subarachnoid hemorrhage. Lancet Neurol. 2003;2(1):12.

3. Phillips LH, Whisnant JP, O'Fallon WM, et al. The unchanging pattern of subarachnoid hemorrhage in a community. Neurology. 1980;30(10):1034 1040 .

4. Davis JM, Hesselink JR. Vascular lesions:intracerebral hemorrhage. In:Taveras JM, Ferrucci JT (Eds.), Radiology: diagnosis imaging intervention. Philadelphia: Lippincott; 1988. p. 1-15.

5. Cloft HJ, Joseph GJ, Dion JE. Risk of cerebral angiography in patients with subarachnoid hemorrhage, cerebral aneurysms and arteriovenous malformations:a meta analysis. Stroke. 1999;30(2):317-320.

6. Liang EY, Chan M, Hsiang JH, et al. Detection and assessment of intracranial aneurysms; Value of CT angiography with shaded surface display. AJR Am J Roentgenol. 1995;165(6):1497-1502.

7. Kato Y, Sano H, Katada K, et al. Application of three dimensional computed tomographic angiography to cerebral aneurysms. Surg Neurol. 1999;52(2):113-121.
8. Ogawa T, Okudera T, Noguchi K, et al. Cerebral aneurysms:evaluation with three dimensional CT angiography. AJNR Am J Neuroradiol. 1996;17(3):447-453

9. Bonita R, Thomson S. Subarachnoid hemorrhage epidemiology, diagnosis, management and outcome. Stroke. 1985;16(4):591-594

10. Dorsch NWC, Young N, Kingston RJ, et al. Early experience with spiral CT in the diagnosis of intracranial aneurysms. Neurosurgery. 1995;36(1):230-238.

11. Ljunggren B, Saveland H, Brandt L, et al. Aneurysymal subarachnoid hemorrhage. Total annual outcome in 1.46 million population. Surg Neurol. 1984;22(5):435-438.

12. Schmid UD, Steiger HJ, Huber P. Accuracy of high resolution computed tomography in direct diagnosis of cerebral aneurysms. Neuroradiology. 1990;29(2):152-159.

13. Katada K, Anno H, Koga S, et al. Three dimensional angiography with helical scanning computed tomography. Radiology. 1990;177(Suppl):364.

14. Scheartz RB, Tice HM, Hooten SM, et al. Evaluation of Cerebral aneurysms with helical computed tomographic correlation with conventional angiography and MR angiography. Radiology. 1994;192(3):717-722.

15. Hoh BL, Cheung AC, Rabinov JD, et al. Results of a prospective protocol of computed tomographic angiography as the only diagnostic and pretreatment planning study for cerebral aneurysms by a combined neurovascular team. Neurosurgery. 2004;54(6):1329-1340.

16. Kassell NF, Tomer JC, Haley EC, et al. The international study on the timing of aneurysm surgery Part 1:Overall management results. $J$ Neurosurg. 1990;73(1):18-36.

17. Pechlivanis I, Schmeidler K, Scholz M, et al. 3-dimensional CT angiography for use of surgery planning in patients with intracranial aneurysms. Acta Neurochir. 2005;147(10):1045-1053.

18. Rosenom J, Eskesen V, Schmidt K. Clinical features and out come in females and males with ruptured intracranial sacular aneurysms. $\mathrm{Br}$ Neurosurg. 1993;7(3):287-290.

19. Laidlaw JD, Sui KH. Ultra-early surgery for aneurysmal subarachnoid hemorrhage:outcome for a consecutive series of 391 patient not selected by grade or age. J Neurosurg. 2002;97(2):250-258.

20. Inagawa T, Takechi A, Yahara K, et al. Primary intracerebral and aneurysmal subarachnoid hemorrhage in Izumo City, Japan. Part I:incidence and seasonal and diurnal variations. J Neurosurg. 2000;93(6):958-966.

21. Sundt TM, Kobayashi S, Fode NC, et al. Results and complications of surgical management of 809 intracranial aneurysms in 722 cases, related and unrelated to grade of patients, type of aneurysym and timing of surgery. J Neurosurg. 1982;56(6):753-765.

22. Socco RL, Wolf PA, Bharucha NE, et al. (1984) Subarachnoid and intra cranial hemorrhage:natural history, prognosis and precursive factors in the Framingham study. Neurology. 1984;34(7):847-854.

23. Ender G, Kagstorm E, Wallsted L. Total over all management and surgical outcome after aneurysmal subarachnoid hemorrhage in a defined population. Br J Neurosurg. 1992;6(5):490-420.

24. Le Roux PD, Dailey AT, Newell DW, et al. Emergent aneurysm clipping without angiography in moribund patient with intracerebral hemorrhage:The use of infusion computed tomographic scans. Neurosurgery. 1993;33(2):189-197.

25. Uysal E, Yanbuloglu B, Erturk M, et al. Spiral CT angiography in diagnosis of cerebral aneurysms of cases with acute subarachnoid hemorrhage. Diagn Interv Radiol. 2005;11(2):77-82.

26. Korogi Y, Takahashi M, Katada K, et al. Intracranial aneurysms:Detection with three dimensional CTA with volume rendering-comparison with conventional angiographic and surgical findings. Radiology. 1999;211(2):497-506. 
27. Nyil K, Nath BR, Suresh N, et al. Middle Cerebral Artery Aneurysms, An Institutional Experience in a South Indian Population. Neuro Surg $Q$. 2008;18(40):246-250.

28. Matsumoto M, Endo $\mathrm{Y}$, Sato M, et al. Acute aneurysm surgery using three dimensional CT angiography without conventional catheter angiography. Fukushima J Med Sci. 2002;48(2):63-73.

29. Heffez DS, Mikhael M, Jensen K. Operative confirmation of threedimensional computed tomographic \& magnetic resonance imaging of cerebrova scular pathology. J Image Guid Surg. 1995;1(3):179-190.
30. Boet R, Poon WS, Lam JM, et al. The surgical treatment of intracranial aneurysms based on computed tomographic angiography alonestreamlining the acute management of symptomatic aneurysms. Acta Neurochir (Wein). 2003;145(2):101-105.

31. Zouaoui A, Sahel M, Marro B, et al. Three-dimensional computed tomographic angiography in detection of cerebral aneurysms in acute subarachnoid hemorrhage. Neurosurgery. 1997;41(1):125-130.

32. Matsumoto M, Satosh M, Nakano M, et al. Three dimensional computed tomography angiography-guided surgery of acutely ruptured cerebral aneurysms. J Neurosurg. 2001;94(5):718-727. 